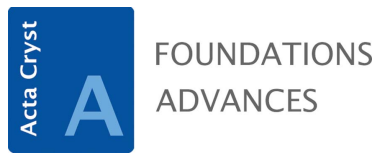

ISSN 2053-2733
Keywords: book review; molecular symmetry; spectroscopy

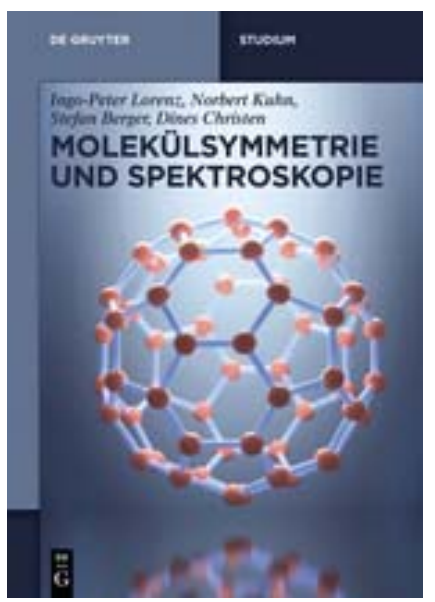

\section{Molekülsymmetrie und Spektroskopie. By Ingo-Peter Lorenz, Norbert Kuhn, Stefan Berger and Dines Christen. De Gruyter, 2015. Paperback, Pp. xvi +244 . Price EUR 49.95/USD 70.00/GBP 37.99. ISBN 978-3-11-036492-7.}

\author{
Dominik Schaniel*
}

Université de Lorraine, CRM2, UMR 7036, Vandoeuvre-les-Nancy, F-54506, France, and CNRS, CRM2, UMR 7036, Vandoeuvre-les-Nancy, F-54506, France. *Correspondence e-mail: dominik.schaniel@univ-lorraine.fr

The book is intended for students of chemistry in order to familiarize themselves with the concepts of molecular symmetry, symmetry operations and point groups. These fundamentals are then applied to vibrational, electronic and NMR spectroscopy. The book is organized into ten chapters. Following a short introduction, Chapter 2 covers the essentials of symmetry elements and symmetry operations and Chapter 3 deals with the theory of point groups. Both chapters are illustrated with many useful examples, so that the concepts can be internalized. The general approach in the book is to treat the theoretical foundations in a very compact form and to rely on the detailed development of examples to lay out the concepts. Further, additional examples are treated as exercises with the solutions given at the end of the book. This requires readers either to be familiar with the underlying mathematics or to work it out by themselves, the focus being on physical content.

Chapters 4 and 5 treat the representation of groups and the corresponding character tables. Again, many examples are treated in detail. Here, the reader should not be afraid of the compactness of the mathematical formalism. By working through the examples, a practical introduction to the use of these powerful tools is offered. Chapter 6 quickly deals with external and internal coordinates. Chapter 7 is dedicated to the application of the concepts of group theory and symmetry to vibrational spectroscopy; at various occasions useful practical advice in the form of 'recipes' is given. At the end of the chapter molecular vibrations are treated in the context of space groups and the crystal lattice. Chapter 8 introduces the notions of the electronic structure of the free ion. It is followed by a description of the ion in the ligand field and the corresponding term diagrams and selection rules in Chapter 9. Chapter 10 treats the application of symmetry rules in NMR spectroscopy. It is less extensive than the preceding chapters on vibrational and electronic spectroscopy. Appendix $A$ lists the character tables, Appendix $B$ the 'Abzähltabellen', Appendix $C$ the Tabane-Sugano diagrams and Appendix $D$ the solutions to the exercises.

In conclusion, the book Molekülsymmetrie und Spektroskopie offers the student (mastering the German language) a compact introduction to the basic concepts via a variety of detailed examples. The book contains very few typographical errors, which is remarkable given the plethora of tables and figures containing symmetry-related symbols. I recommend this book if one is willing to work through the examples. 